\title{
Quantitative Analysis of DEPES Distributions of Pt(111)
}

\author{
K. KRUK* AND I. MORAWSKI \\ Institute of Experimental Physics, University of Wrocław \\ pl. M. Borna 9, 50-204 Wrocław, Poland \\ Received: 20.02.2020 \& Accepted: 24.06.2020 \\ Doi: 10.12693/APhysPolA.138.459 \\ *e-mail: karolina.kruk@ifd.uni.wroc.pl
}

\begin{abstract}
Directional elastic peak electron spectroscopy (DEPES) was used to reveal a short range order within the near-surface region of $\mathrm{Pt}(111)$. Experiments were performed at primary electron beam energies in the range from $0.9 \mathrm{keV}$ to $1.8 \mathrm{keV}$. DEPES signal was recorded for a wide range of polar $\theta$ and azimuthal $\phi$ angles and then transformed to two-dimensional DEPES maps using stereographic projection. Theoretical DEPES distributions were obtained with the application of the multiple scattering (MS) theory. In MS calculations, the following parameters were varied: the inelastic mean free path $\lambda$, the radius around the emitter $R_{\max }$ and the cones of smoothing $M$. The comparison of experimental and theoretical DEPES maps was performed by an $R$-factor analysis in order to find the best correspondence between the recorded and calculated distributions. The absolute minimum of the $R$-factor was found at rather small values of the radius around the emitter and large inelastic mean free paths. However, the obtained minimum was very shallow and took the form of a characteristic band in the $R_{\max }(l)$ plots. Thus, other $R_{\max }$ and $\lambda$ values corresponding to the local $R$-factor minimum can also be to some extent used in computations. The cone of smoothing, which reflects the spread of primary beam directions before it strikes the sample, decreases at larger electron energies which scales with better electron focusing at larger $E_{p}$ values. The discussion concerns the computation time associated with the mentioned parameters and the convergence of theoretical data.
\end{abstract}

topics: retarding field analyser (RFA), directional elastic peak electron spectroscopy (DEPES), crystalline structure, short range order, scattering of primary electrons

\section{Introduction}

The directional elastic peak electron spectroscopy (DEPES) [1-4] is an experimental method which utilizes the phenomenon of elastic scattering of primary electrons striking the sample on atoms in a crystalline solid at electron energies above $500 \mathrm{eV}$. At these medium electron energies the forward scattering effect $[5,6]$ occurs which leads to the anisotropic distribution of scattered electrons. As a consequence, the flux of electrons in solids after scattering is mainly focused along the direction of the electron beam propagation. This effect is used to reveal the structure within the near surface region of crystalline samples [4]. The limited value of the inelastic mean free path of primary and emitted electrons as well as the defocusing effect [7-9] of primaries reduce the information depth of the crystalline structure to the first few atomic layers.

Forward scattering is a universal phenomenon which concerns all electrons independently on the direction of their propagation, including primary electrons striking the crystalline samples but also secondary electrons emitted from solids. Therefore, it is also used to record angular distributions of photo- and Auger electrons in X-ray photoelectron diffraction (XPD) and Auger electron diffraction (AED) [10-14], respectively. In XPD and AED a small aperture hemispherical analyzer (HA) $[15,16]$ is used which enables the recording of secondary electrons emitted in a certain direction. In the case of DEPES, however, the measurements are carried out with the application of a large acceptance angle analyzer such as a retarding field analyser (RFA) [17], usually applied for low-energy electron diffraction (LEED) [18-20] pattern observations and Auger electron spectroscopy (AES) $[21,22]$ measurements. In this case, angular distribution of the electron emission is integrated over a large collector, therefore the recorded signal is mainly effected by the primary beam electron scattering effects. Furthermore, the recorded intensities depend on the electron energy, inelastic mean free path and the kind of atoms in investigated solids.

The scattering process of electrons in solids is described by different theoretical approximations [23-26]. In calculations, different parameters are involved in order to reflect a real experimental and scattering geometry, electron attenuation, scattering properties of atoms and weights of emitters. The resulting theoretical data are compared to experimental results by means of an $R$-factor analysis which enables finding quantitative information about the sample [4, 27]. 
In this work, the analysis of experimental and theoretical DEPES data for the $\mathrm{Pt}(111)$ surface is presented. In computations, the wave field in a solid was calculated with the use of the multiple scattering (MS) approximation [26]. The calculations were carried out by varying three parameters involved in the description of the scattering process of primary electrons on the atomic potential, namely: inelastic mean free path $\lambda$, the radius around the emitter $R_{\max }$, and the cone of smoothing $M$. The considered parameters are associated with the real scattering geometry in the sample and influence the calculated intensities. The inelastic mean free path describes the attenuation of the electron wave in a solid. The radius around the emitter indicates a number of scattering atoms, which considerably influence the final wave field at the emitter site. Both parameters are, therefore, critical for the time of calculations. The cone of smoothing takes into account the angular distribution of the incident electron beam just after leaving the electron gun. Therefore, this parameter reflects the focusing of the electron beam just before it strikes the sample. The experimental and theoretical DEPES distributions were compared by means of a $R$-factor analysis in order to find the best correspondence between theory and experiment. The computation of DEPES theoretical distributions is discussed in the context of the used parameters.

\section{Experiment}

The investigations were carried out for a $\mathrm{Pt}(111)$ monocrystal. The ultra-high vacuum (UHV) chamber was equipped with a retarding field analyser (RFA) with an acceptance angle equal to $110^{\circ}$ and a manipulator, which enabled the precise azimuthal and polar rotation of a sample with respect to the axial electron gun of RFA. In the UHV system, the pressure was lower than $10^{-8} \mathrm{~Pa}$. The $\operatorname{Pt}(111)$ was cleaned using $\mathrm{Ar}^{+}$ion sputtering, annealing at the temperature around $1500 \mathrm{~K}$ and flashing. The cleanness of the surface was controlled by AES, and a long range order of surface atoms was monitored by LEED. In DEPES measurements, the primary electron beam with fixed energy $E_{p}$ in the range of 900-1800 eV struck the crystalline sample and the current of elastically backscattered electrons was measured during the sample rotation. The recording of elastically backscattered electrons was performed in the $N(E)$ mode of the RFA operation. During the measurements of the elastic peak height, the sample was rotated around the polar axis (parallel to the surface sample) at a fixed azimuthal angle, which was chosen by the rotation of the sample around the azimuthal axis perpendicular to the sample surface. During DEPES investigations, the polar angle $\theta$ was changed from $-80^{\circ}$ to $+80^{\circ}$ with increments of $\Delta \theta=0.25^{\circ}$ and the azimuthal angle $\varphi$ was altered in the range of $0^{\circ}-180^{\circ}$ in $\Delta \varphi=2^{\circ}$ steps. The set of DEPES polar profiles recorded at different azimuthal angles

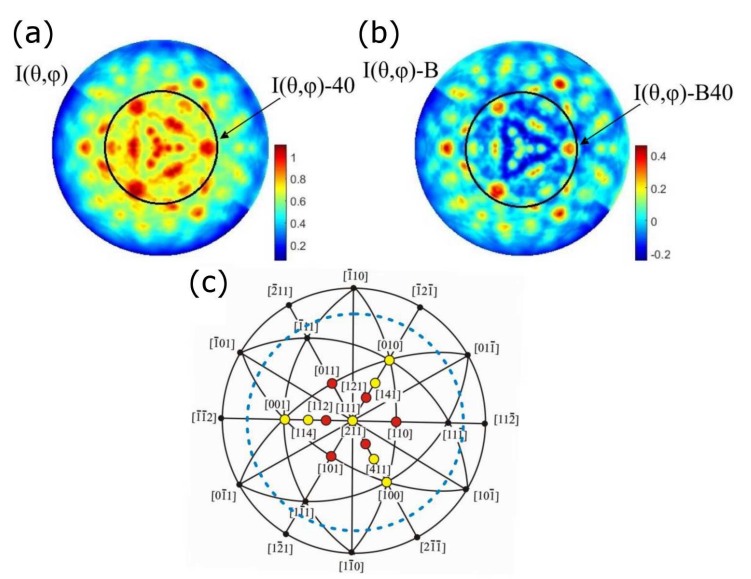

Fig. 1. (a) Experimental DEPES map $I(\theta, \phi)$ recorded for $\mathrm{Pt}(111)$ at the primary electron beam energy $E_{p}=1200 \mathrm{eV}$ and the range of polar angles between $-80^{\circ}$ and $80^{\circ}$. The circle surrounds DEPES intensities within the polar angle range from $-40^{\circ}$ to $40^{\circ}$, which is denoted as $I(\theta, \phi)-40$. (b) Experimental DEPES distribution after the background subtraction $I(\theta, \phi)$-B. As in (a), the area inside the circle ranges from $-40^{\circ}$ to $40^{\circ}$, which is denoted as $I(\theta, \phi)$-B40. (c) Stereographic projection of a (111) terminated fcc monocrystal. The area surrounded by the dashed line indicates incidence beam directions available experimentally ( $\theta$ ranges from $-80^{\circ}$ to $80^{\circ}$ ) used in (a) and (b). The contrast for experimental $I(\theta, \phi)$ and $I(\theta, \phi)-40$ maps is equal to 1.77 and 0.68 , respectively.

was transformed to a two-dimensional DEPES map presented as a stereographic projection. The angular coordinates $(\theta, \varphi)$ were transformed to Cartesian coordinates $(x, y)$ according to the relation: $x=\tan (\theta / 2) \sin (\varphi))$ and $y=2 \tan (\theta / 2) \cos (\varphi)$. For each map the intensities were reflected by the colour scale. The background subtraction was made with the use of the cosine function of the incidence angle $\theta$ as $I_{B G}=A \cos (B \theta)$, where $A$ and $B$ are parameters fitted by means of the least square analysis [28]. This function was found to reflect the background intensities of mono- and polycrystalline samples in the best way [4]. In the data evaluation, fragments of experimental and theoretical results published in $[29,30]$ were used.

\section{Results and discussion}

The DEPES map recorded for the primary electron beam energy $E_{p}=1200 \mathrm{eV}$ and the wide polar angle range from $-80^{\circ}$ to $80^{\circ}$ before and after the background subtraction [28], denoted as $I(\theta, \phi)$ and $I(\theta, \phi)-B$, respectively, is shown in Fig. 1. The signal strength from the minimum to maximum value is reflected by the attached colour scale. A part of the map within the polar angle range between $-40^{\circ}$ and $40^{\circ}$ surrounded by the black circle is denoted as $I(\theta, \phi)-40$ and $I(\theta, \phi)$-B40 for DEPES intensities before and after the background subtraction, 
respectively. In this way four maps for each energy $E_{p}$ were considered for further evaluation. The intensity maxima observed in the experimental DEPES map correspond to the distribution of closepacked atomic rows in a (111) face-centred cubic (fcc) monocrystal.

Theoretical DEPES maps were calculated taking into account the crystalline structure of the $\mathrm{Pt}(111)$ sample, phase shifts obtained with the use of the muffin-tin approximation [18], electron energies and associated inelastic mean free path $(\lambda)$ values [31-33], the multiple scattering (MS) events of primary electrons [26], the radius around the emitter $\left(R_{\max }\right)$ and the cone of smoothing $(M)$ [4,34]. In calculations the number of elastic scattering events of electrons on atomic potential was limited to 3 which assures the sufficient convergence of theoretical data $[4,35]$. The comparison of experimental and theoretical DEPES data as well as two theoretical DEPES distributions calculated for different computation parameters was made by the $R$-factor analysis. In the latter case, the convergence of theoretical data can be determined. In the quantitative comparison of experimental and theoretical DEPES maps, the following $R$-factor based on the Pearson correlation coefficient $[4,29,34]$ was used:

$$
R=1-\frac{\sum_{i=1}^{N}\left(I_{\mathrm{ex}_{i}}-\bar{I}_{\mathrm{ex}}\right)\left(I_{\mathrm{th}_{i}}-\bar{I}_{\mathrm{th}}\right)}{\sqrt{\sum_{i=1}^{N}\left(I_{\mathrm{ex}_{i}}-\bar{I}_{\mathrm{ex}}\right)^{2} \sum_{i=1}^{N}\left(I_{\mathrm{th}_{i}}-\bar{I}_{\mathrm{th}}\right)^{2}}}
$$

where $I_{\mathrm{ex}}$ and $I_{\mathrm{th}}$ are the experimental and theoretical signals, respectively, while $i$ numerates the measured and calculated $(\theta, \phi)$ points of the DEPES stereographic distribution and $N$ is the number of points for the entire map. The quantitative analysis of two theoretical DEPES maps involves respective theoretical intensities in (1).

The minimum of the $R$-factor indicates the best correspondence between experimental and theoretical results. In Fig. 2 the $R$-factor obtained for two theoretical DEPES maps calculated at subsequent $n$ and $n+1$ cluster layers and different primary electron beam energies $E_{p}$ is shown as a function of the number of sample layers taken into account in MS calculations. A better convergence of theoretical results is observed with the increase of the number of the considered layers. As expected, at higher electron energies more layers should be considered in MS calculations to obtain the same $R$-factor values which scales with larger inelastic mean free paths. No change of $R$-factor values at a larger number of considered layers indicates no differences between theoretical DEPES distributions obtained at the same $E_{p}$.

In MS calculations for a given primary electron beam energy, the values of the inelastic mean free path and the radius around the emitter were varied. In Fig. 3 the decimal logarithm of $t$ is shown as a function of the multiplier $l$ of the inelastic mean

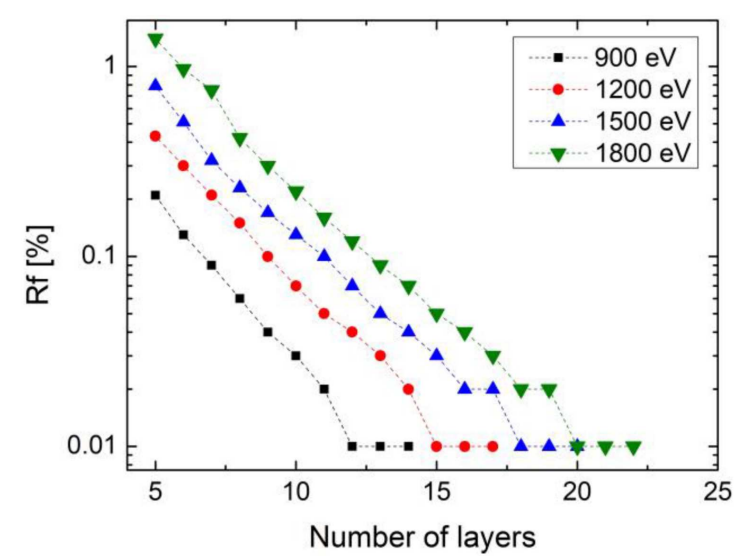

Fig. 2. The $R$-factor as a function of the number of layers in $\mathrm{Pt}(111)$ obtained for two theoretical DEPES maps calculated at subsequent $n$ and $n+1$ cluster layers and $E_{p}$ equal to $900 \mathrm{eV}, 1200 \mathrm{eV}, 1500 \mathrm{eV}$, and $1800 \mathrm{eV}$. The $R$-factor is shown for a larger $n+1$ considered number of layers.
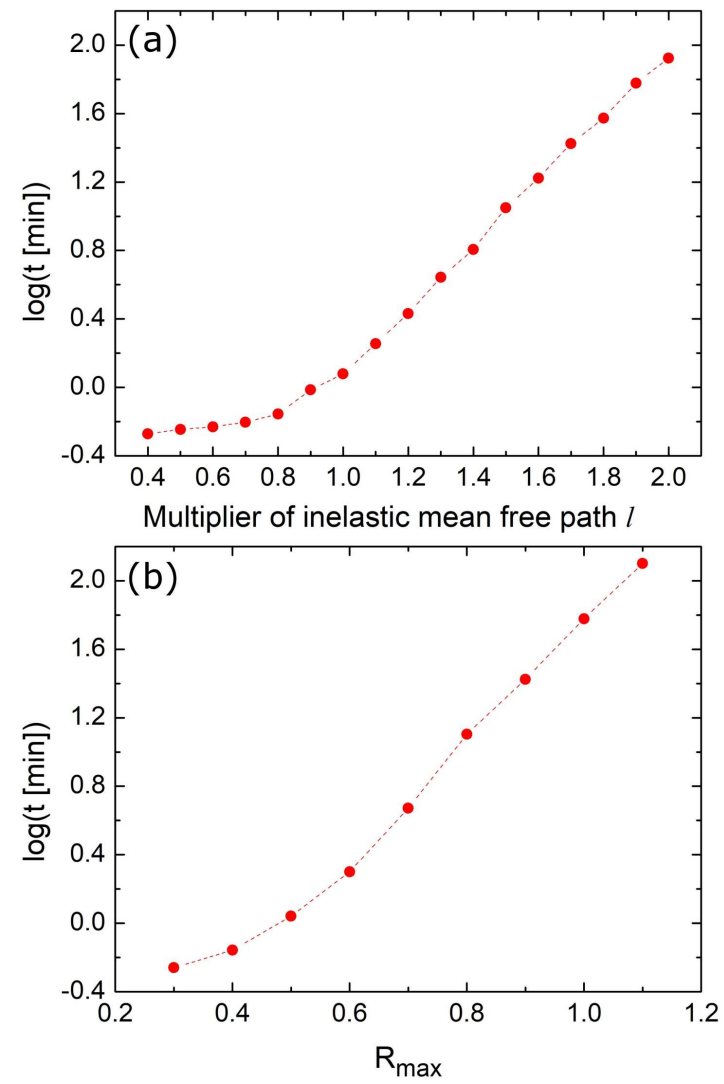

Fig. 3. The logarithm of the calculation time of a theoretical DEPES map at $E_{p}=1200 \mathrm{eV}$ and constant $R_{\max }=0.9$ as a function of the multiplier $l$ of the inelastic mean free path (a) and the radius around the emitter at constant $l=1.7(\mathrm{~b})$.

free path $\lambda$ at constant $R_{\max }=0.9$ and the radius around the emitter $R_{\max }$ at constant $l=1.7$. As expected, the considerable increase of the computation time is observed with the increase of $l$ and $R_{\max }$. Both computation parameters scale with the primary electron beam energy in experiment. 
The classical interpretation of the inelastic mean free path considers $\lambda$ as an average distance measured along the trajectory of a particle at a given energy between two inelastic collisions in a matter. In the case of DEPES, the incident electron beam striking the crystalline sample interacts with an atomic potential which results in elastic or inelastic scattering events. In the quantum mechanical description of the scattering process, the primary electrons are described by a plane wave and the scattered electrons by spherical waves emanating from the scattering centres located on the atomic cores [4]. The elastic scattering leads to the constructive or destructive interference of primary and scattered waves resulting in a wave field in a solid. The inelastic scattering events lead to the damping of electrons in a sample. Both elastic and inelastic scattering events were taken into account in the MS calculations by considering appropriate scattering factors [36] and exponential decay of the electron wave amplitude. The calculations were performed at different polar $\theta$ and azimuthal $\phi$ angles which reflect mutual orientations of an axial electron gun and the crystalline sample as well as different wave lengths associated with energies of primary electrons used in the experiment. In the literature, $\lambda$ values were determined basing on: Tanuma-Powell-Penn (TPP-2) predictive formula [37], Gries model [38], EPES (elastic peak electron spectroscopy) results and Monte Carlo calculations [39-42] as well as a model dielectric function [43]. The Bethe equation with some modifications is suggested for electron energies lower than $200 \mathrm{eV}$ in $[33,40]$. The values of $\lambda$ determined with the use of these models for electron energies in the range of 50-2000 eV are particularly useful for analytical methods such as X-ray photoelectron spectroscopy (XPS) and AES.

The dependence of $\lambda$ on the electron energy obtained with the use of the above mentioned approximations is shown in Fig. 4. Similar $\lambda$ values in the considered energy range from $400 \mathrm{eV}$ to $2000 \mathrm{eV}$ arise from TPP2 and Gries models. The EPES measurements and Monte Carlo simulations give significantly lower $\lambda$ values especially at higher electron energies. Because of a large number of applications, theoretical DEPES distributions were calculated taking into account the inelastic mean free paths obtained with the use of the TPP-2 model. Then, at a given electron energy, the $\lambda$ value was varied by the multiplier $l$ in the range from 0.4 to 2.0 with 0.1 steps. The influence of the $\lambda$ value on the DEPES intensities is shown in Fig. 5. The signal modulation within each DEPES map is reflected by the contrast defined as $C=2\left(I_{\max }-I_{\min }\right) /\left(I_{\max }+I_{\min }\right)$, where $I_{\max }$ and $I_{\min }$ are the maximum and minimum intensities. The increase of $\lambda$ results in a more detailed intensity pattern and large contrast values which are associated with the contribution of deeper sample layers to the DEPES intensities. In all DEPES

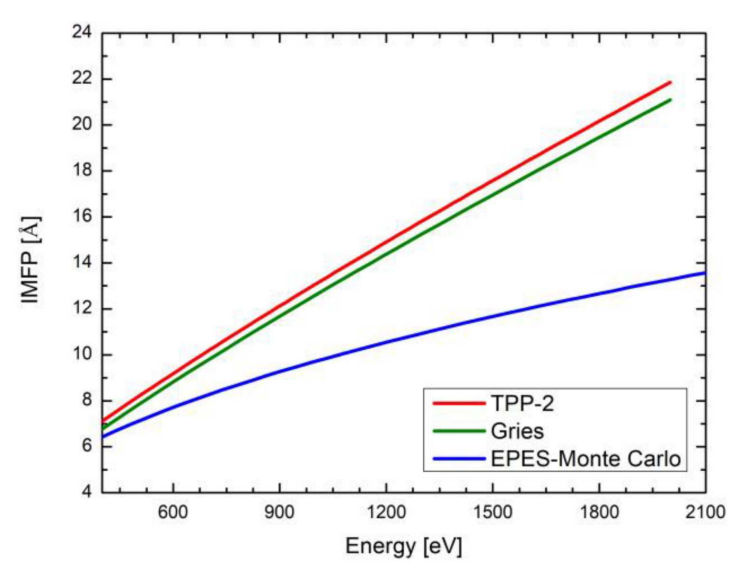

Fig. 4. Inelastic mean free path $\lambda$ as a function of the electron energy for $\mathrm{Pt}$ according to NIST database [40] obtained with the use of the TPP2 [37] and Gries [38] approximations, as well as the EPES measurements and Monte Carlo calculations [39, 41, 42].
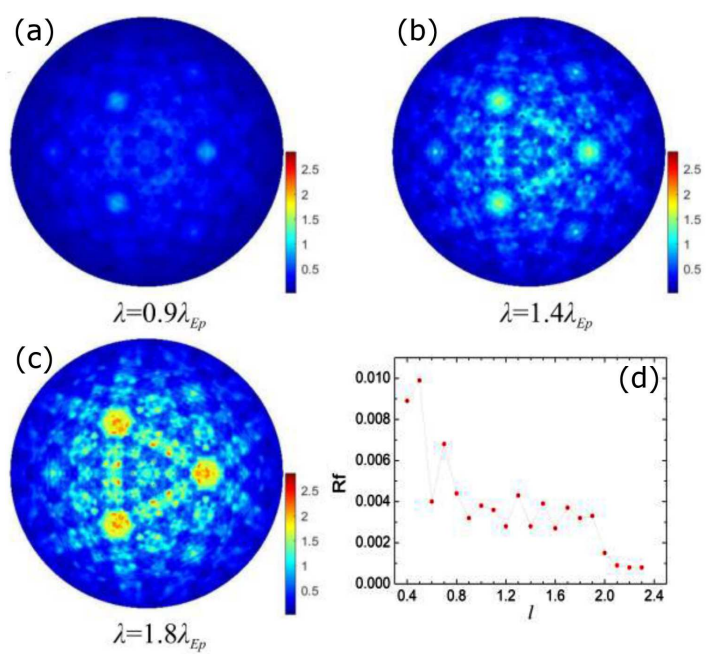

Fig. 5. Theoretical DEPES maps $I(\theta, \phi)$ at the primary electron beam energy $E_{p}=1200 \mathrm{eV}$ calculated for $\mathrm{Pt}(111)$ at $R_{\max }=1.0, M=0.0^{\circ}$ and different multipliers $l$ of the inelastic mean free path of the primary electrons $\lambda_{E_{p}}$ : (a) $l=0.9$, (b) $l=1.4$, and (c) $l=1.8$. (d) The $R$-factor as a function of the multiplier $l$ of $\lambda$ calculated for subsequent pairs of theoretical maps, e.g. for $l=1.1$ and $l=1.2$. Contrast values are equal to: (a) $C=1.91$, (b) $C=1.89$, and (c) $C=1.9$.

distributions, the main intensity maxima associated with close-packed crystallographic directions are well visible. The characteristic intensity bands associated with crystallographic planes are better reflected at larger $l$ values. Experimentally, this effect is achieved at increased primary electron beam energies [30]. At lower $E_{p}$, the intensity DEPES pattern is less detailed and characterised by broad maxima which makes the analysis of the crystalline structure ambiguous. The convergence of theoretical data was proved by the calculation of an $R$-factor for 

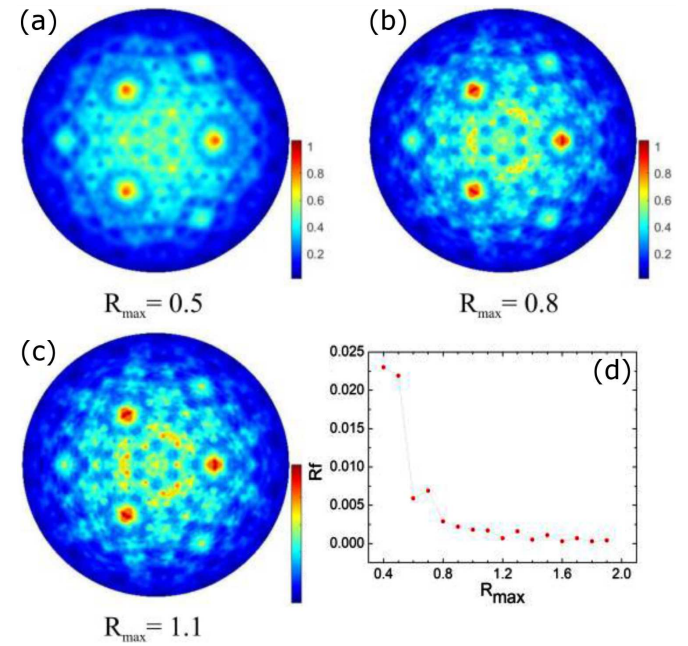

Fig. 6. Theoretical DEPES maps $I(\theta, \phi)$ for $\operatorname{Pt}(111)$ at $1200 \mathrm{eV}, l=1.0, M=0.0^{\circ}$, and different values of the radius around the emitter $R_{\max }$ : (a) 0.5 , (b) 0.8 , and (c) 1.1. (d). The $R$-factor as a function of the radius around the emitter calculated for subsequent pairs of theoretical maps, e.g. for $R_{\max }=0.5$ and $R_{\max }=0.6$. Contrast values are equal to: (a) $C=1.9$, (b) $C=1.9$, and (c) $C=1.93$.

theoretical DEPES distributions obtained at constant $R_{\max }=1.0$ and $M=0.0^{\circ}$ and increased $l$ multipliers of $\lambda$ with respect to the DEPES distribution at $l+0.1$. The $R$-factor falls to 0.001 above $l=2.0$. This means that a subsequent calculation for a larger $l$ does not result in more detailed maps. Also for this range of $l$ the computation time is reasonable (Fig. 3).

The radius around the emitter is a dimensionless geometrical parameter (normalized to the value of the inelastic mean free path at given energy), which limits the number of scatterers of electron wave to the sphere of radius $R_{\max }$ around the considered emitter $[34,35]$. In other words, this parameter reduces the number of scattered waves finally reaching the emitter site. In Fig. 6 the theoretical DEPES maps for $\mathrm{Pt}(111)$ calculated at $1200 \mathrm{eV}, l=1.0$ and $M=0.0^{\circ}$ are shown at different $R_{\max }$ values. The increase of $R_{\max }$ results in a more detailed intensity pattern and larger contrast because of the contribution of a larger number of scattered waves to the DEPES signal. We proved the convergence of theoretical results by an $R$-factor analysis. $R$-factor was obtained for theoretical DEPES maps calculated at increased $R_{\max }$ with respect to the DEPES distribution obtained at $R_{\max }=2.4$. As expected, the decrease of $R$-factor observed with $R_{\max }$ indicates a better convergence of theoretical data. The $R$ factor values show that $R_{\max }$ above about 1.5 do not result in significantly more detailed intensity patterns in DEPES maps. Therefore, the consideration of $R_{\max }$ up to about 1.5 assures the sufficient convergence of theoretical data. Nevertheless, due to computation time $R_{\max }$ was limited to 1.1 (Fig. 3).
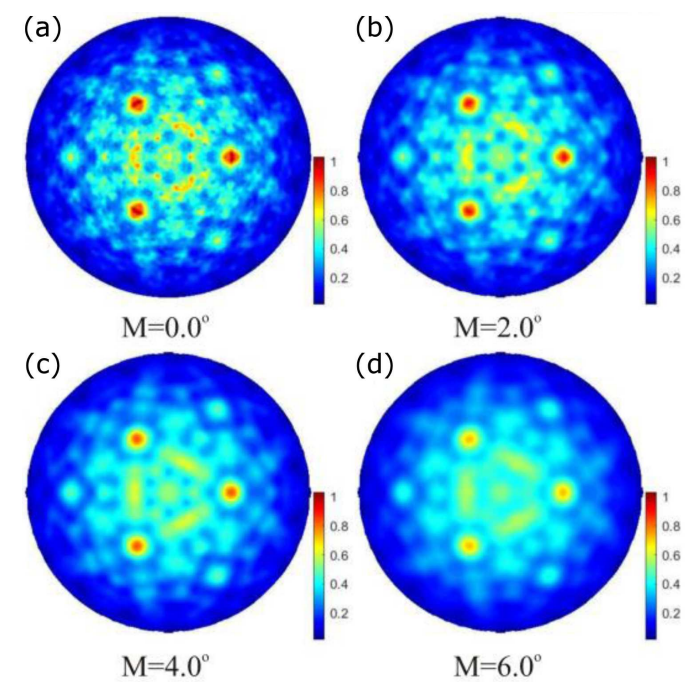

Fig. 7. Theoretical DEPES maps $I(\theta, \phi)$ for $\mathrm{Pt}(111)$ at $1200 \mathrm{eV}, l=1.0$, and $R_{\max }=1.0$ calculated at different smoothing cones $M$ : (a) $0.0^{\circ}$, (b) $2.0^{\circ}$, (c) $4.0^{\circ}$, and (d) $6.0^{\circ}$. Contrast values are equal to: (a) $C=1.92$, (b) $C=1.88$, (c) $C=1.86$, and (d) $C=1.83$.

Another computation parameter taken into account in MS calculations is the cone of smoothing $M$ which reflects the geometry of the incidence electron beam. In experiment, the primary beam is characterised by a dispersion angle which results from repulsive interactions of electrons and their focusing in the electron gun. Therefore, the primary electrons - just after leaving the electron gun form a cone with a small but not negligible angle. As it was shown in [34], the dispersion angle equals a few degrees and decreases with the electron beam energy. In MS calculations, the electron beam is considered to be a plane wave with a well-defined propagation direction. In order to take into account the above mentioned effects the cone of smoothing was introduced [4, 34]. The DEPES intensities are averaged within a small (a few degrees) cone around the incidence direction which corresponds to a circle of a certain radius (smoothing radius)on a stereographic plot. In calculations, the cone of smoothing was considered to vary in the range from $0.0^{\circ}$ to $6.0^{\circ}$ with $0.5^{\circ}$ increments around the incidence beam direction. Because of the integration of the DEPES signal within a small cone around the incidence direction — which reflects a number of incidence beam directions in experiment - less details of the intensity pattern and lower contrast values are observed with the increase of $M$ (Fig. 7).

A further analysis concerns the calculation of $R$-factor for experimental and theoretical DEPES data, the latter obtained by changing the $l, R_{\max }$, and $M$ parameters. The result of such an analysis for the $I(\theta, \phi)$-B distribution at $1500 \mathrm{eV}, M=3.0^{\circ}$ and different $l$ and $R_{\max }$ values is shown in Fig. 8 . The range of the $R$-factor values, reflected by 


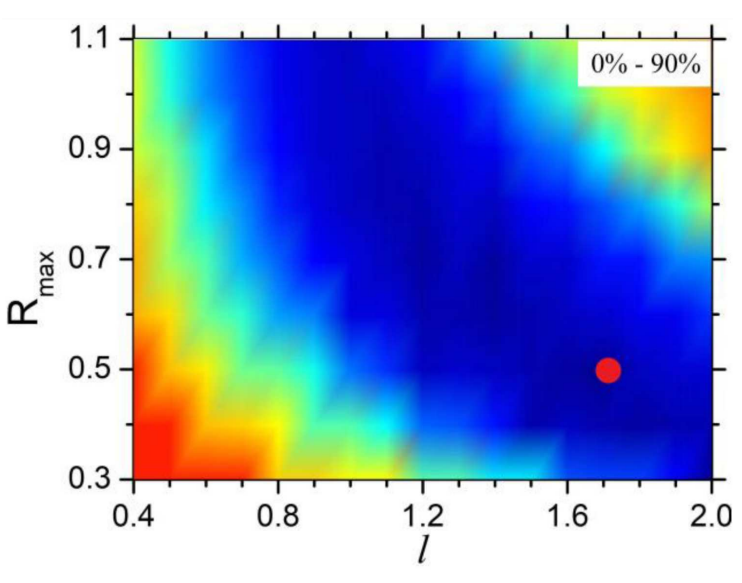

Fig. 8. The $R$-factor calculated for experimental and theoretical $I(\theta, \phi)$-B distributions at $1500 \mathrm{eV}$. Theoretical DEPES data were obtained at $M=3.0^{\circ}$ and different $l$ (from 0.4 to 2.0) and $R_{\max }$ (from 0.3 to 1.1 ) values. The absolute minimum of the $R$-factor is marked by the red dot. The variation of an $R$-factor, given in percent in the upper right corner with respect to the highest value shown in this work, is reflected by colour.

colour, within the plot is given in percent with respect to the highest value shown in this work. For small and large $l$ and $R_{\max }$, the $R$-factor achieves large values which indicates a large discrepancy between theoretical and experimental DEPES distributions. The characteristic band of low $R$-factor values, indicating the correspondence between experimental and theoretical data, is observed with increased $l$ and associated decrease of $R_{\max }$. The absolute minimum of the $R$-factor equal to 0.1159 , marked by the red circle, was found at $l=1.7$ and $R_{\max }=0.5$. For example, at $l=1.0$ and $R_{\max }=1.0$ the $R$-factor minimum equals 0.1250 which shows small differences between $R$-factors obtained within the characteristic band in Fig. 8. The similar plot of $R$-factor as a function of $l$ and $R_{\max }$ was found for all other electron energies.

The $R$-factor analysis was made for experimental and theoretical $I(\theta, \phi)$ distributions obtained at different primary electron beam energies $E_{p}$. The experimental results were compared to theoretical data, the latter calculated at different $M, l$ and $R_{\max }$. The results of this analysis are shown in Fig. 9. As in Fig. 8, the similar distribution of $R$-factor values is observed including their rise at small and large $l$ and $R_{\max }$ as well as their decrease taking a form of a broad band with a hyperbolelike shape. It stems from the fact that both $R_{\max }$ and $l$ limit the number of scattered wave functions, however in the latter case this limitation is done by decreasing the wave function amplitude in an exponential manner. In almost all cases, the absolute minimum of the $R$-factor was found at large $l$ and small $R_{\max }$. This minimum is, however, very shallow as it is also visible in Fig. 8. Therefore, other local minima visible in Figs. 8 and 9, taking a form of a characteristic band, also reflect a good correspondence between experimental and theoretical data. The increase of $E_{p}$ at constant $M$ leads to larger $R$-factor values, especially at low and high $l$ and $R_{\max }$.

The $R$-factor analysis of experimental and theoretical DEPES distributions for a wide polar angle range $I(\theta, \phi)$, limited to the maximal polar angle $40^{\circ}$ denoted as $I(\theta, \phi)-40$, after the background subtraction $I(\theta, \phi)$-B as well as limited to the maximal polar angle $40^{\circ}$ and after the subtraction of the background $I(\theta, \phi)$-B40 at $1500 \mathrm{eV}$ as a function of $M, l$, and $R_{\max }$ is shown in Fig. 10. The data indicate a similar distribution of $R$-factor values as in Figs. 8 and 9 independently on the applied background subtraction procedure and the limitation of the maximal polar angle to $40^{\circ}$. The absolute minimum of the $R$-factor is observed in almost all cases at similar $l$ and $R_{\max }$, which confirms a very shallow minimum of the $R$-factor values. Moreover, at the same primary electron beam energy $E_{p}=1.2 \mathrm{keV}$ the lowest values of the $R$-factors are noted for a set of data obtained at the cone of smoothing $M=2^{\circ}$, which scales with previous observations [34]. This confirms that at this energy the incident beam directions spread within a few degrees which results in the averaging and smoothing of experimental DEPES intensities.

In Fig. 11 the absolute minimum of the $R$-factor read out from the $R_{\max }(l)$ plots (as shown in Figs. 8-10) is presented as a function of the cone of smoothing $M$ for experimental and theoretical $I(\theta, \phi), I(\theta, \phi)-40, I(\theta, \phi)-\mathrm{B}$, and $I(\theta, \phi)$ B40 DEPES distributions at the primary electron beam energy $E_{p}$ equal to $900 \mathrm{eV}$ and $1500 \mathrm{eV}$. The pronounced minimum for all considered DEPES maps is observed, which reflects the best agreement between experimental and theoretical data. The $R$-factor minimum is independent of the kind of DEPES map considered in the analysis at $E_{p}=900 \mathrm{eV}$. At $E_{p}=1500 \mathrm{eV}$, however, the $R$-factor minimum is observed at slightly different $M$ values. Generally, the characteristic shift of the $R$-factor minimum towards lower $M$ values with $E_{p}$ (Fig. 11a and b) is noted, which is associated with a better focusing of the primary electron beam in the electron gun at larger $E_{p}$ values. This tendency is well reflected in Fig. 11c, where the smoothing cone associated with the $R$-factor minimum is shown as a function of $E_{p}$ for all considered DEPES distributions. Such an analysis allows to determine the distribution of incidence angles of primaries before they strike the sample and gives a direct evidence of the focusing of the primary electron beam in the electron gun of the RFA analyzer.

In Fig. 12 experimental DEPES distributions $I(\theta, \phi)$ recorded at the primary electron beam energy $900 \mathrm{eV}$ and $1500 \mathrm{eV}$ are shown together with the results of calculations at $l=1.0, R_{\max }=1.0$, and $M=0.0^{\circ}$ as well as at the best fit. 
M
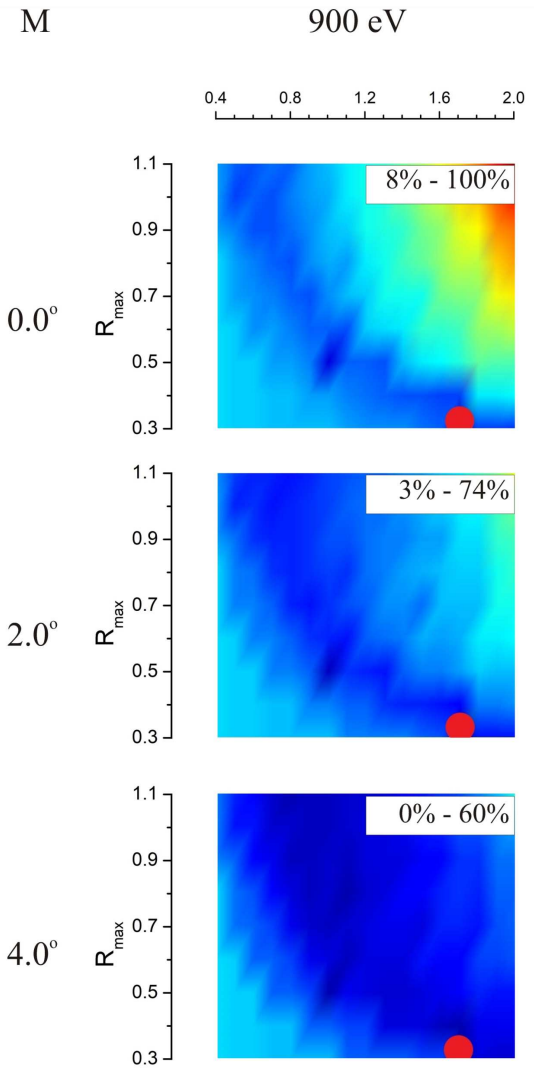

$6.0^{\circ}$
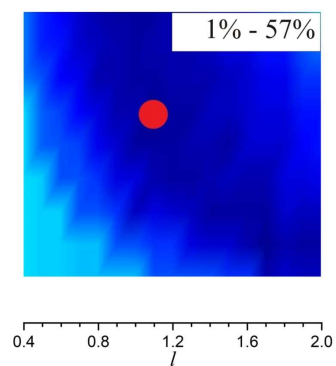

$1200 \mathrm{eV}$

$1800 \mathrm{eV}$
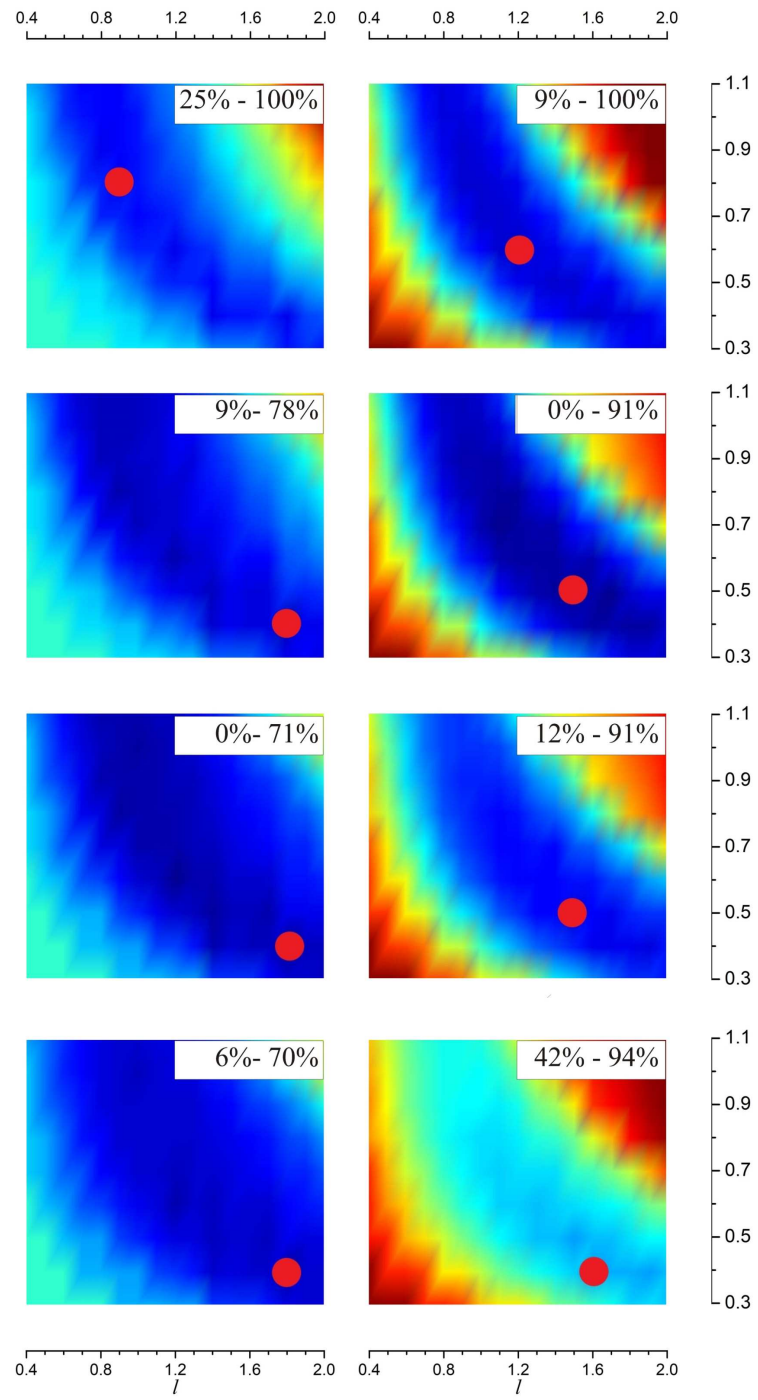

Fig. 9. The $R$-factor calculated for experimental and theoretical distributions $I(\theta, \phi)$ at $900 \mathrm{eV}, 1200 \mathrm{eV}$, and $1800 \mathrm{eV}$. Theoretical DEPES data were obtained at $M$ equal to $0.0^{\circ}, 2.0^{\circ}, 4.0^{\circ}$ and $6.0^{\circ}$ as a function of $l$ (from 0.4 to 2.0 ) and $R_{\max }$ (from 0.3 to 1.1). The absolute minimum of the $R$-factor is marked by the red circle. The range of the $R$-factor, given in the upper right corner as a percentage of the highest obtained value, is reflected by colour.

TABLE I

The $R$-factor minimum $\left(R f_{\min }\right)$ obtained for experimental and theoretical $I(\theta, \phi)$ and $I(\theta, \phi)-40$ DEPES maps at different primary electron beam energies $E_{p}$ and associated $l, R_{\max }$ and $M$ parameters of calculations. Contrast and $R$-factor (Rf) values at $l=1.0, R_{\max }=1.0$ and $M=0.0^{\circ}$.

\begin{tabular}{|c|c|c|c|c|c|c|c|c|c|c|}
\hline & $E_{p}[\mathrm{eV}]$ & $C$ & $M\left[{ }^{\circ}\right]$ & $l$ & $\lambda[\AA]$ & $R_{\max }$ & $R f_{\min }$ & $C$ & $\begin{array}{c}R f \text { for } l=1.0, \\
R_{\max }=1.0, M=0^{\circ}\end{array}$ & $C$ \\
\hline \multirow{4}{*}{$\frac{2}{2}$} & 900 & 1.65 & 3.5 & 1.7 & 21 & 0.3 & 0.8830 & 1.82 & 0.1396 & 1.92 \\
\hline & 1200 & 1.77 & 4.0 & 1.8 & 27 & 0.4 & 0.7790 & 1.87 & 0.1069 & 1.92 \\
\hline & 1500 & 1.61 & 3.0 & 1.7 & 30 & 0.5 & 0.1159 & 1.86 & 0.1361 & 1.93 \\
\hline & 1800 & 1.28 & 2.0 & 1.5 & 30 & 0.5 & 0.1350 & 1.91 & 0.1572 & 1.93 \\
\hline \multirow{4}{*}{$\begin{array}{l}\stackrel{0}{\not} \\
\frac{1}{1} \\
\frac{1}{2} \\
\stackrel{0}{0}\end{array}$} & 900 & 0.65 & 4.5 & 1.1 & 13 & 0.8 & 0.2520 & 0.91 & 0.3451 & 1.28 \\
\hline & 1200 & 0.68 & 2.0 & 1.8 & 27 & 0.4 & 0.1857 & 1.10 & 0.2364 & 1.27 \\
\hline & 1500 & 0.75 & 1.5 & 1.4 & 25 & 0.8 & 0.2244 & 1.26 & 0.2474 & 1.36 \\
\hline & 1800 & 0.91 & 1.5 & 1.7 & 34 & 0.5 & 0.2268 & 1.50 & 0.2538 & 1.54 \\
\hline
\end{tabular}


M<smiles>C1CCCCC1</smiles>

$\mathrm{I}(\theta, \varphi)$

$\mathrm{I}(\theta, \varphi)-40$

$\mathrm{I}(\theta, \varphi)-\mathrm{B}$

$\mathrm{I}(\theta, \varphi)-\mathrm{B} 40$

$0.0^{\circ}$ $0.4 \quad 0.8 \quad 1.2 \quad 1.6 \quad 2.0$
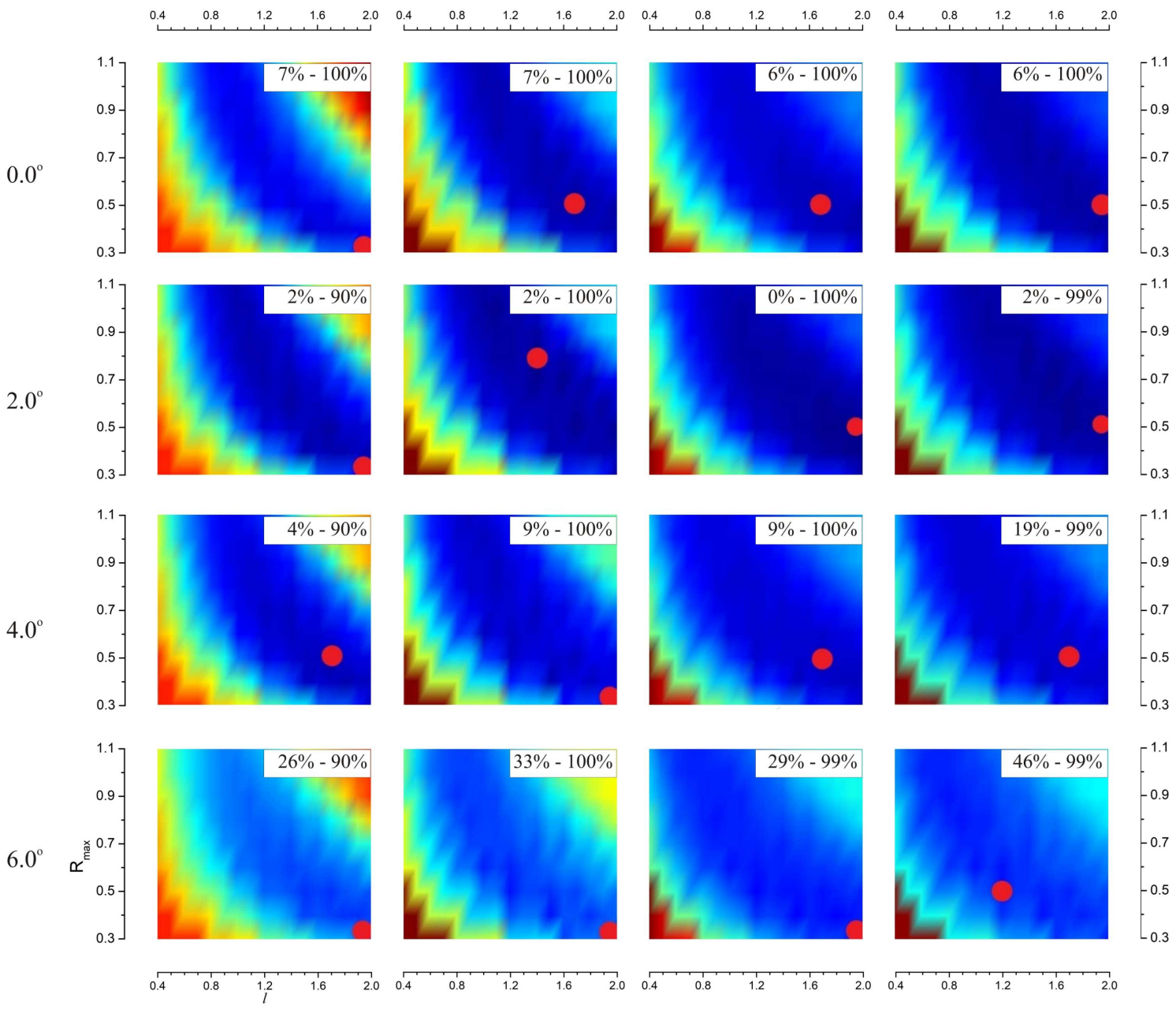

Fig. 10. The $R$-factor calculated for experimental and theoretical DEPES distributions $I(\theta, \phi), I(\theta, \phi)-40$ $I(\theta, \phi)-\mathrm{B}$, and $I(\theta, \phi)-\mathrm{B} 40$ at $1500 \mathrm{eV}$. Theoretical DEPES data were obtained at $M$ equal to $0.0^{\circ}, 2.0^{\circ}, 4.0^{\circ}$ and $6.0^{\circ}$ as a function of $l$ (from 0.4 to 2.0 ) and $R_{\max }$ (from 0.3 to 1.1). The absolute $R$-factor minimum is denoted by the red circle. The range of the $R$-factor shown as a percentage of the highest obtained value in the upper right corner is scaled by colour.

As in Table I but for $I(\theta, \phi)$-B, and $I(\theta, \phi)$-B40 DEPES distributions.

TABLE II

\begin{tabular}{|c|c|c|c|c|c|c|c|}
\hline & $E_{p}[\mathrm{eV}]$ & $M\left[{ }^{\circ}\right]$ & $l$ & $\lambda[\AA]$ & $R_{\text {max }}$ & $R f_{\min }$ & $\begin{array}{c}R f \text { for } l=1.0, \\
R_{\max }=1.0, M=0^{\circ}\end{array}$ \\
\hline \multirow{4}{*}{$\begin{array}{l}\frac{n}{2} \\
\frac{1}{2} \\
=\end{array}$} & 900 & 3.5 & 1.8 & 22 & 0.7 & 0.3757 & 0.4936 \\
\hline & 1200 & 2.0 & 1.7 & 25 & 0.6 & 0.2563 & 0.3237 \\
\hline & 1500 & 1.5 & 2.0 & 35 & 0.5 & 0.2455 & 0.2920 \\
\hline & 1800 & 1.5 & 1.7 & 34 & 0.5 & 0.2450 & 0.2920 \\
\hline \multirow{4}{*}{$\begin{array}{l}\stackrel{0}{\not} \\
\frac{1}{1} \\
\frac{1}{\theta} \\
\stackrel{0}{0}\end{array}$} & 900 & 3.5 & 1.5 & 18 & 0.7 & 0.2858 & 0.3981 \\
\hline & 1200 & 2.0 & 1.7 & 25 & 0.6 & 0.1910 & 0.2528 \\
\hline & 1500 & 1.5 & 2.0 & 35 & 0.5 & 0.2201 & 0.2726 \\
\hline & 1800 & 1.5 & 1.7 & 34 & 0.5 & 0.2256 & 0.2570 \\
\hline
\end{tabular}

$R$-factor minimum $\left(\mathrm{Rf}_{\min }\right)$ obtained for experimental and theoretical $I(\theta, \phi), I(\theta, \phi)-40, I(\theta, \phi)-\mathrm{B}$, and $I(\theta, \phi)$-B40 DEPES maps at different primary electron beam energies $E_{p}$ and associated $l$, $R_{\text {max }}$, and $M$ parameters as well as contrast and
$R$-factor $(\mathrm{Rf})$ values at $l=1.0, R_{\max }=1.0$, and $M=0.0^{\circ}$ are summarised in Tables I and II. Generally, the best fit between experimental and theoretical DEPES data was found for rather large $l$ and small $R_{\max }$ values. 

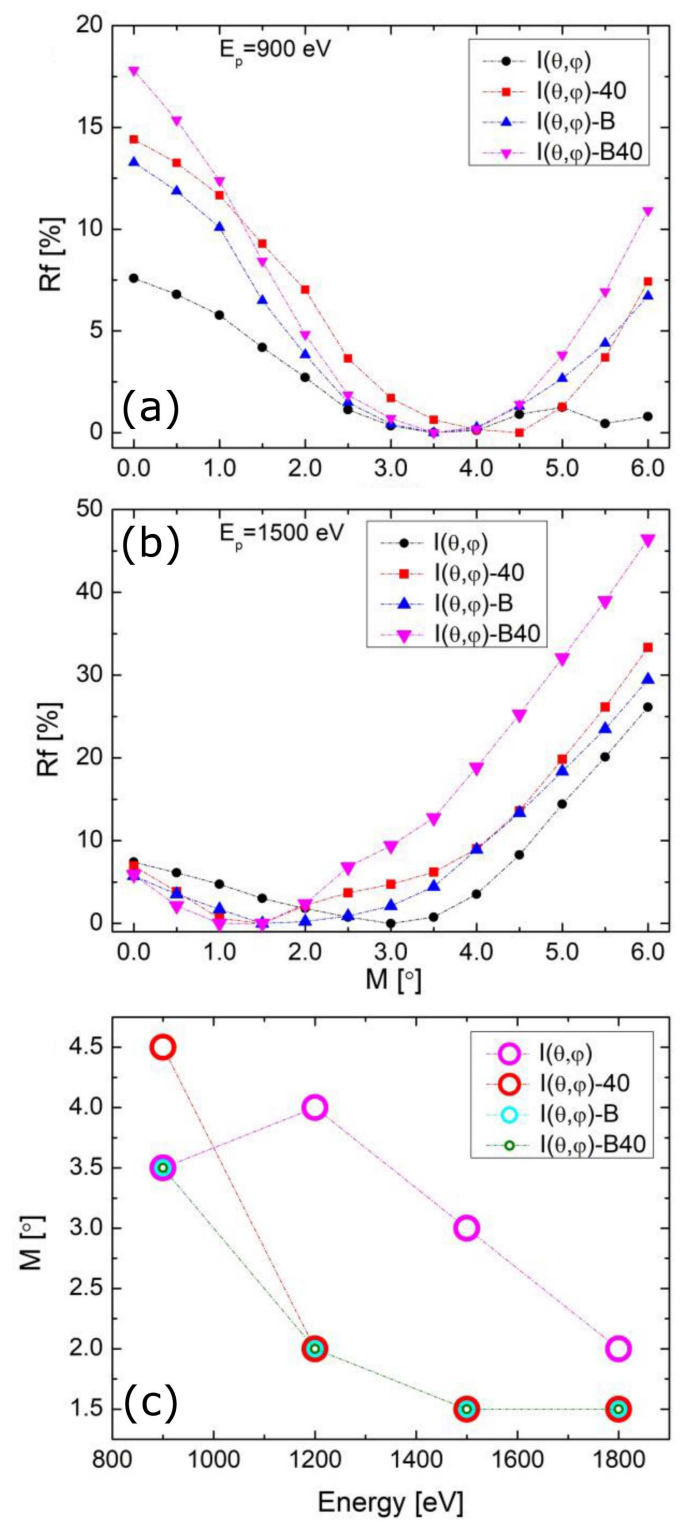

Fig. 11. The minimum value of the $R$-factor (Rf) obtained from the $R_{\max }(l)$ plots (as shown in Figs. 8-10) as a function of the cone of smoothing $M$ for experimental and theoretical DEPES maps $I(\theta, \phi), I(\theta, \phi)-40, I(\theta, \phi)$-B and $I(\theta, \phi)-\mathrm{B} 40$ at the primary electron beam energy $E_{p}$ equal to $900 \mathrm{eV}$ (a) and $1500 \mathrm{eV}$ (b). The smoothing cone corresponding to the $R$-factor absolute minimum for all kinds of DEPES maps as a function of $E_{p}(\mathrm{c})$.

The results presented in this paper prove that the application of DEPES allows to reflect a short range order within the first few atomic layers of monocrystalline samples. Due to the forward scattering of the collimated primary electron beam the characteristic intensity maxima observed in the DEPES stereographic distributions reflect the Pt(111) sample crystallinity. The simulations performed with the use of the MS approximation varying the inelastic mean free path $\lambda$ as well as different geometrical parameters enabled finding the best fit of theoretical

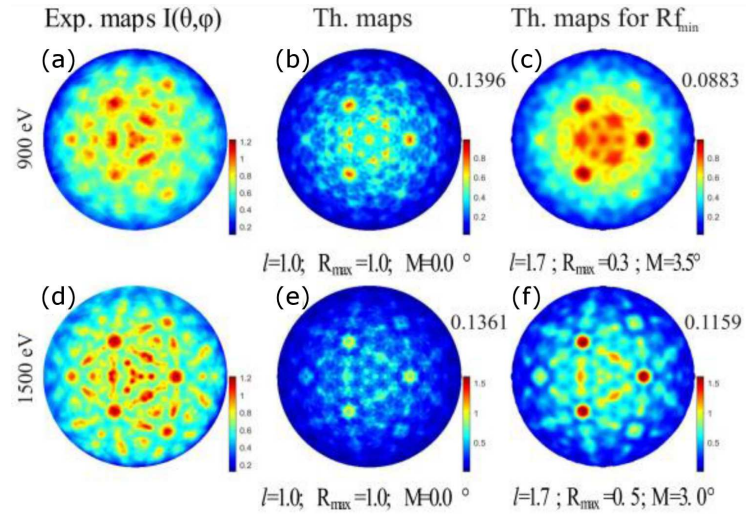

Fig. 12. Experimental DEPES distributions $I(\theta, \phi)$ recorded at $E_{p}=900 \mathrm{eV}$ (a) and $E_{p}=1500 \mathrm{eV}(\mathrm{d})$. Theoretical DEPES maps $I(\theta, \phi)$ calculated at $l=1.0, R_{\max }=1.0, M=0.0^{\circ}$ and $E_{p}=900 \mathrm{eV}$ (b) and $E_{p}=1500 \mathrm{eV}$ (e). Theoretical DEPES data $I(\theta, \phi)$ obtained at the best fit with the experimental distributions ( $R$-factor minimum) calculated at $l=1.7, R_{\max }=0.3$ and $M=3.5^{\circ}$ at $E_{p}=900 \mathrm{eV}$ (c) and $l=1.7$, $R_{\max }=0.5$, and $M=3.0^{\circ}$ at $E_{p}=150 \mathrm{eV}$ (f). The $R$-factor values are indicated in the upper right corner of theoretical distributions. Contrast values are equal to: (a) $C=1.65$, (b) $C=1.92$, (c) $C=1.82$, (d) $C=1.61,($ e) $C=1.93$ and (f) $C=1.86$.

results to the experimental DEPES data. This quantitative analysis results in $l, R_{\max }, M$ and $\lambda$ values which determine the signal measured in electron spectroscopy methods such as DEPES.

\section{Conclusions}

In this work the experimental DEPES distributions were compared to the theoretical DEPES maps obtained with the use of different computation parameters such as the inelastic mean free path, the radius around the emitter and the cone of smoothing. The analysis of the DEPES results enabled finding the short range order within the near surface region of $\mathrm{Pt}(111)$. The data obtained at different primary electron beam energies $E_{p}$ show that the best agreement between theory and experiment was found for small $R_{\max }$ and large $\lambda$ values. However, the $R$-factor minimum is very shallow. Therefore, other $R_{\max }$ and $\lambda$ values associated with the local $R$-factor minimum also - to some extent - reflect the correspondence between theory and experiment. The cone of smoothing associated with the focusing of primary electrons decreases with increasing electron energy $E_{p}$. This reflects the spread of primary beam directions before it strikes the sample and shows better focusing at larger $E_{p}$. The quantitative analysis performed with the use of the $R$-factor enables to determine the number of layers and the radius around the emitter taken in calculations at different $E_{p}$ which assure the sufficient convergence of theoretical data and the reasonable computation time of theoretical DEPES distributions. 


\section{Acknowledgments}

The University of Wrocław is acknowledged for the financial support 1010/S/IFD. The calculations were carried out in the Wroclaw Centre for Networking and Supercomputing (www.wcss.pl).

\section{References}

[1] S. Mróz, M. Nowicki, Surf. Sci. 297, 66 (1993).

[2] S. Mróz, Prog. Surf. Sci. 48, 157 (1995).

[3] M. Nowicki, Vacuum 54, 73 (1999).

[4] I. Morawski, M. Nowicki, Surf. Sci. Rep. 74, 178 (2019).

[5] H. Li, S.Y. Tong, Surf. Sci. Lett. 281 , L347 (1993).

[6] H. C. Poon, S. Y. Tong, Phys. Rev. B 30, 6211 (1984).

[7] M.L. Xu, J.J. Barton, M.A. Van Hove, Phys. Rev. B 39, 8275 (1989).

[8] S.Y. Tong, H.C. Poon, D.R. Snider, Phys. Rev. B 32, 2096 (1985).

[9] W.F. Egelhoff Jr., Phys. Rev. Lett. 59, 559 (1987).

[10] C.S. Fadley, S. Kono, L.G. Petersson, S.M. Goldberg, N.F.T. Hall, J.T. Lloyd, Z. Hussain, Surf. Sci. 89, 52 (1979).

[11] C.S. Fadley, Prog. Surf. Sci. 16, 275 (1984).

[12] S.A. Chambers, Adv. Phys. 40, 357 (1991).

[13] S.A. Chambers, Surf. Sci. Rep. 16, 261 (1992).

[14] W.F. Egelhoff Jr., in: Ultrathin Magnetic Structures I. An introduction to electronic, magnetic, and structural properties, Eds. J.A.C. Bland, B. Heinrich, Springer Verlag, Berlin 1994, p. 220.

[15] J. Osterwalder, in: Surface and Interface Analysis: Concepts and Methods, Eds. K. Wandelt, Wiley, Berlin 2012, p. 151.

[16] M.P. Seah, G.C. Smith, M.T. Anthony, Surf. Inter. Anal. 15, 293 (1990).

[17] R.E. Weber, W. T. Peria, J. Appl. Phys, 38, 4355 (1967).

[18] J. Pendry, Low energy electron diffraction: The theory and its Application to Determination of Surface Structure, Academic Press, London, 1974.

[19] M.A. Van Hove, W.H. Weinberg, C.M. Chan, Low-Energy Electron Diffraction: Experiment, Theory and Surface Structure Determination, Springer Verlag, Berlin, 1986.

[20] K. Heinz, in Surface and Interface Analysis: Concepts and Methods, Eds. K. Wandelt, Wiley, Berlin 2012, p. 93.
[21] M. Thompson, M.D. Baker, A. Christie, J.F. Tyson, Auger electron spectroscopy, Wiley, New York 1985.

[22] K.D. Childs, B.A. Carlson, L.A. LaVanier, J.F. Moulder, D.F. Paul, W.F. Stickle, D.G. Watson, Handbook of Auger Electron Spectroscopy, 3rd edition, Physical Electronics Inc., Eden Prairie (MN) 1995.

[23] P. Morin, Surf. Sci. 164, 127 (1985).

[24] S.K. Andersen, A. Howie, Surf. Sci. 50, 197 (1975).

[25] A. Stuck, M. Nowicki, S. Mróz, D. Naumović, J. Osterwalder, Surf. Sci. 306, 21 (1994).

[26] I. Morawski, M. Nowicki, Phys. Rev. B 75, 155412 (2007).

[27] M. Nowicki, Phys. Rev. B 69, 245421 (2004).

[28] I. Morawski, M. Nowicki,J. El. Spect. Rel. Phenom. 163, 65 (2008).

[29] A. Miszczuk, I. Morawski, R. Kucharczyk, M. Nowicki, Appl. Surf. Sci. 373, 32 (2016).

[30] I. Morawski, M. Nowicki, J. Elec. Spec. Rel. Phen. 185, 90 (2012).

[31] M.P. Seah, W.A. Dench, Surf. Inter. Anal. 1, 2 (1979).

[32] S. Tanuma, C.J. Powell, D.R. Penn, Surf. Int. Anal. 17, 911 (1991).

[33] C.J. Powell, A. Jablonski, J. Phys. Chem. Ref. Data 28, 19 (1999).

[34] M. Jurczyszyn, A. Miszczuk, I. Morawski, I. Zasada, M. Nowicki, Mat. Charact. 93 , 94 (2014).

[35] I. Morawski, Ph.D. Thesis, University of Wrocław, 2008.

[36] J.J. Rehr, R.C. Alberts, Phys. Rev. B 41, 8139 (1990).

[37] S. Tanuma, C.J. Powell, D.R. Penn, Surf. Interf. Anal. 43, 689 (2011).

[38] W.H. Gries, Surf. Inter. Anal. 24, 38 (1996).

[39] G. Gergely, S. Gurban, M. Menyhard, A. Jablonski, L. Zommer, Acta Phys. Pol. A 114, S49 (2008).

[40] C.J. Powell, A. Jablonski, NIST Electron Inelastic-Mean-Free-Path Database, Ver. 1.2, National Institute of Standards and Technology, Gaithersburg (MD) 2010.

[41] A. Jablonski, Surf. Sci. 151, 166 (1985).

[42] A. Jablonski, J. Gryko, J. Kraaer, S. Tougaard, Phys. Rev. B 39, 61 (1989).

[43] D.R. Penn, Phys. Rev. B 35, 482 (1987). 